\title{
A novel mutation in SIRT1-AS leading to a decreased risk of HCC
}

\author{
JUNBIN LIU ${ }^{1}$, WEIHONG WU ${ }^{2}$ and JUNHUA JIN ${ }^{1}$ \\ ${ }^{1}$ Department of Hepatobiliary Surgery, The Affiliated Hospital of Inner Mongolia Medical College; \\ ${ }^{2}$ Outpatient Office of The Affiliated Hospital of Inner Mongolia Medical College, Hohhot, Inner Mongolia, P.R. China
}

Received May 11, 2015; Accepted June 23, 2015

DOI: 10.3892/or.2015.4205

\begin{abstract}
Natural antisense transcripts (NATs) have recently been associated with the development of human cancers. However, the interrelationship between NATs and their sense transcripts in hepatocellular carcinoma (HCC) has not been well-characterized. The aim of the present study was to characterize the AS IncRNA SIRT1-AS, which suppressed the miRNA-induced translational repression of SIRT1 mRNA by masking the miR-29c binding site on the SIRT1 3'UTR. A 763-nucleotide (nt), single-exon NAT transcribed from the antisense strand of SIRT1 gene, designated as SIRT1-AS, was identified using strand-specific RT-PCR and northern blot analyses. SIRT1-AS overexpression promoted the proliferation of the human HCC cell lines by upregulating the SIRT1 protein level. The mechanism was that SIRT1-AS bound to SIRT1 mRNA at 3'UTR, masked the miR-29c binding site and stabilized SIRT1 mRNA. A single-nucleotide mutation $(622 \mathrm{U}>\mathrm{C})$ in the SIRT1-AS sequence was found when we used gene sequencing as an assistant approach for HCC diagnosis. Bioinformatics and the RNase protection assay revealed that the mutation led to a marked alteration in the secondary structure of SIRT1-AS and caused its inability to bind with SIRT1 mRNA. Overexpression of this mutant did not have a promoting effect on HCC cell proliferation. Moreover, the mean level of SIRT1-AS was much higher in the HCC patients compared to non-hepatopathy volunteers $(\mathrm{P}<0.01)$, whereas the opposite effect was observed for the mutant ratio. The odds ratio (OR) analysis also suggested the $622 \mathrm{C}$ mutation reduced the risk of HCC. Taken together, we identified a novel mutation in SIRT1-AS leading to a decreased risk of HCC. The results of the present study suggest that the $622 \mathrm{C}$ mutant of SIRT1 antisense transcript suppresses HCC cell line proliferation, decreases the risk of $\mathrm{HCC}$ and is a potential target for gene therapy.
\end{abstract}

Correspondence to: Dr Junbin Liu, Department of Hepatobiliary Surgery, The Affiliated Hospital of Inner Mongolia Medical College, 1 North Channel Road, Hohhot, Inner Mongolia 010050, P.R. China E-mail: junbinliuinner@163.com

Key words: natural antisense transcript, SIRT1, hepatocellular carcinoma, proliferation, miR-29c binding site

\section{Introduction}

High-throughput approaches have revealed numerous overlapping transcripts. Approximately $50 \%$ of the human genome was found to be bidirectionally transcribed and the transcripts from the opposite strand of a functional gene were denoted natural antisense transcripts (NATs) $(1,2)$. The study of gene regulation by antisense transcription is particularly noteworthy, as their genomic arrangement immediately indicates that they may act on each other. NATs, most of which are antisense long non-coding RNAs (AS IncRNAs), play versatile roles in refined regulation in almost all stages of gene expression, including DNA methylation, transitional interference, RNA splicing, editing, stabilization, localization, and translation (3-6). Recent findings have shown that several AS IncRNAs are dysregulated in human cancers, and their aberrant expression is associated with tumorigenesis or metastasis (7-9). However, compared with sense protein-coding genes, the characteristics of their AS transcripts remain to be determined.

Sirtuins are $\mathrm{NAD}^{+}$-dependent deacetylases and major factors in the response to cellular stresses. The importance of sirtuins is manifested by their roles in several major human disorders, such as cancer, cardiovascular diseases, muscular atrophy and neurodegenerative diseases (10-13). SIRT1 is the best-characterized sirtuin in the seven-member family (SIRT1-7), which is involved in cell survival, the cellular stress response, energy metabolism and cell apoptosis (14-16). Silencing SIRT1 in cancer cell lines has been reported to restrain cancer cell proliferation and result in cell cycle arrest and apoptosis (17). Dozens of substrates of SIRT1 have been identified and well-characterized, including p53, p73, FOXO3a, PGC-1 $\alpha$ and PPAR (18). There has been a focus on ncRNAs, which modulate SIRT1 expression and function in the development and progression of human cancer. It has been demonstrated that human miR-29c suppresses SIRT1 by binding to the 3'UTR region of SIRT1 causing translational inhibition in hepatocellular carcinoma (HCC) cells (19). Other ncRNAs such as miR-34a, miR-195, miR-132 and Let-7g were also proven to target the SIRTI gene. However, to the best of our knowledge, no report identifying that lncRNA selectively regulates SIRT1 is available.

In the present study, we identified a single-exon NAT transcribed from the oncogenic SIRT1 antisense strand, designated as SIRT1-AS. SIRT1-AS masked the miR-29c binding site on the SIRT1 3'UTR and stabilized SIRT1 mRNA. Wild-type SIRT1-AS had a promoting effect on HCC cell proliferation in vitro. A single-nucleotide mutation $(622 \mathrm{U}>\mathrm{C})$ was identified 
in SIRT1-AS, which caused its inability to stabilize SIRT1 mRNA and decreased the risk of HCC.

\section{Materials and methods}

Cell culture. The HCC-9903 cell line was presented by Professor Liu Chunsheng from the Qilu Hospital of Shandong University Bioengineering Center (Shandong, China). The cells were taken from liquid nitrogen and then thawed in a $37^{\circ} \mathrm{C}$ water bath. After centrifugation at $1,000 \mathrm{x}$ g for $5 \mathrm{~min}$, the cells were suspended in RPMI-1640 complemented with 10\% FBS (Invitrogen-Life Technologies, Carlsbad, CA, USA) and seeded in 6-well plates at a density of $1 \times 10^{5} / \mathrm{cm}^{2}$.

Strand-specific RT-PCR and $q P C R$. Extracted RNA was treated with DNase I (Fermentas, Thermo Fisher Scientific, Waltham, MA, USA) to exclude the possibility of DNA contamination. The RNA concentration in each sample was quantified using a spectrophotometer at $260 \mathrm{~nm}$ and the purity of RNA was assessed by measuring the OD260/280 ratio (1.85-1.96). The integrity of RNA was checked by electrophoresis on a $1.0 \%$ agarose gel with ethidium bromide staining. Subsequently, $2 \mu \mathrm{g}$ of DNase I-treated RNA was applied in the reverse transcription reaction with $2 \mathrm{pM}$ specific RT primer and SuperScript III reverse transcriptase (Invitrogen-Life Technologies). PCR reactions were performed on $1 \mu 1$ of strand-specific cDNA by mixing $2 X$ Thermo Pol Taq buffer (New England Biolabs, Ipswich, MA, USA), $0.25 \mathrm{mM}$ forward primer, $0.25 \mathrm{mM}$ reverse primer, $0.2 \mathrm{mM}$ dNTPs (Amersham, Buckinghamshire, UK), and 1.25 units Taq polymerase (New England Biolabs). The PCR conditions used were: $95^{\circ} \mathrm{C}$ for 5 min followed by 30 cycles of $95^{\circ} \mathrm{C}$ for $30 \mathrm{sec}, 48^{\circ} \mathrm{C}$ for $30 \mathrm{sec}$ and $72^{\circ} \mathrm{C}$ for $45 \mathrm{sec}$ with a final incubation at $72^{\circ} \mathrm{C}$ for $10 \mathrm{~min}$. The product was checked by electrophoresis on a $1.5 \%$ agarose gel with ethidium bromide staining.

RT-qPCR reactions were carried out in a final volume of $25 \mu$ l, using SYBR Premix Ex Taq (Takara), $0.4 \mathrm{mM}$ of each primer, and $200 \mathrm{ng}$ of cDNA template. Each individual sample was run in triplicate wells. PCR amplification cycles were performed using the $\mathrm{iQ}^{\mathrm{TM}} 5$ Multicolor Real-Time PCR detection system (Bio-Rad, Hercules, CA, USA) and SYBR Premix Ex Taq II kit (Takara, Dalian, China). The reactions were initially denatured at $95^{\circ} \mathrm{C}$ for $3 \mathrm{~min}$ followed by 40 cycles of $95^{\circ} \mathrm{C}$ for $10 \mathrm{sec}, 50^{\circ} \mathrm{C}$ for $30 \mathrm{sec}, 72^{\circ} \mathrm{C}$ for $20 \mathrm{sec}$. The change in transcript abundance of all tested genes was calculated using the $2^{-\Delta \Delta C t}$ method. Gene mRNA amounts were normalized to $\beta$-actin.

Northern blotting. Northern blotting was performed according to a previous study (?). Specific hybridization probe complementary to SIRT1-AS was designed and synthesized by GenScript Co. (Nanjing, China). Total RNA $(20 \mu \mathrm{g})$ was analyzed on a $12 \%$ polyacrylamide denaturing gel containing 7.5 $\mathrm{M}$ urea and transferred onto a Hybond $\mathrm{N}^{+}$nylon membrane (Amersham). The membranes were cross-linked using ultraviolet light for $30 \mathrm{sec}$ at $1,200 \mathrm{~mJ} / \mathrm{cm}^{2}$. Hybridization was performed according to the manufacturer's instructions. Following washing, the membranes were exposed for 20-40 h to Kodak XAR5 films (Sigma-Aldrich, St. Louis, MO, USA). As a control, the membranes were hybridized with a human U6 snRNA probe. The probes used were: SIRT1-AS, 5'-TAA
CACCAAATCCTCCCAAT-3'; SIRT1 V1, 5'-AGGCAGTTG GAAGATGGCGGACGAGG-3'; SIRT1 V2, 5'-CCTGAGGTT GAGGGCGGCTGGG-3'; and U6, 5'-GCAGGGGCCATG CTAATCTTCT CTGTATCG-3'.

Cell proliferation and viability testing. Proliferation of HCC-9903 was determined using an Apollo ${ }^{\circledR} 567$ EdU staining kit (Roche, Basel, Switzerland) according to the manufacturer's instructions, and the nuclei were stained by Hoechst 33342.

Cell viability was performed using a CellTiter-Blue $\mathrm{H}$ cell viability assay kit (Promega, Promega, WI, USA) according to the manufacturer's instructions.

Plasmid construction and transfection. The full-length cds sequence of SIRT1 mRNA, WT SIRT1-AS and the mutant SIRT1-AS were amplified by PCR using primers containing $K p n I$ (in forward primer) and $\mathrm{XhoI}$ (in reverse primer) sites. The primers used were: SIRT1 forward, 5'-CGGGGTACC TATGCTATGAACAATGGAAG-3' and reverse, 5'-CCGCTC GAGTTGCCTGTTGAGGATTTGGT-3'; and SIRT1-AS forward, 5'-CGGGGTACCAGTCAAATGACAATTTTA ATAGAC-3' and reverse, 5'-CCGCTCGAGTTAGTGCCT GCCTGGA-3'. The products were cleaved and ligated onto corresponding sites of the pcDNA3.1 plasmid, which was confirmed by sequencing. The constructs and miR-29c mimic were transfected with X-tremeGENE DNA transfection reagents (Roche) according to the manufacturer's instructions.

Western blotting. The cells were lysed in lysis buffer (Beyotime Co., Shanghai, China) supplemented with $1 \mathrm{mM} \mathrm{PMSF}$. Protein concentration was determined with the BCA protein assay kit (Tiangen). Protein $(20 \mu \mathrm{g})$ of each sample were separated by 12\% SDS-PAGE and electro-transferred to a PVDF membrane (Millipore, Billerica, MA, USA) for immunoblot analysis. The primary antibodies used were: anti-SIRT1 (1:300), anti-p53 (acetyl K382, 1:200) and anti-p53 (1:400) (all from Abcam, Cambridge, MA, USA), and anti- $\beta$-actin (1:800; Santa Cruz Biotechnology, Inc., Santa Cruz, CA, USA) which was used as the internal reference. After incubation with the appropriate HRP-conjugated secondary antibody, the proteins were detected using a ChemiDoc XRS imaging system and analysis software Quantity One (Bio-Rad).

RNA stability assay. To detect the impact of SIRT1-AS on SIRT1 mRNA half-life, after $48 \mathrm{~h}$ of transfection of each constructs or miR-29c mimic, HCC-9903 cells were treated with $2 \mu \mathrm{g} / \mathrm{ml}$ actinomycin D (Sigma-Aldrich), which had an inhibitory effect on transcription. The cells were collected at $0,0.5,1,2,4,6,8,10$ and $12 \mathrm{~h}$ after treatment, and then total cell RNA was isolated to measure the residual mRNAs by RT-qPCR. GAPDH mRNA was applied as an internal control, which was relatively stable within $32 \mathrm{~h}$.

In vitro ribonuclease protection assay (RPA). According to the sequencing result of SIRT1-AS, full-length WT and mutant SIRT1-AS were synthesized, both of which contained the complimentary sequence of the miR-29c binding site and the no. 622 mutation site. Additionally, $1 \mu \mathrm{g}$ of each fragment was incubated with synthetic SIRT1 mRNA (a part complementary 
A

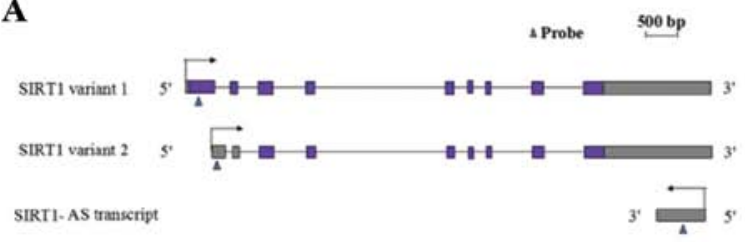

B

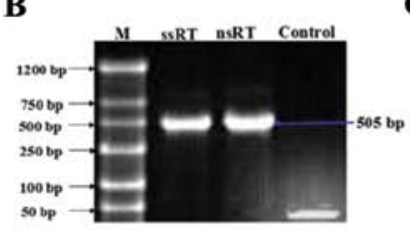

C

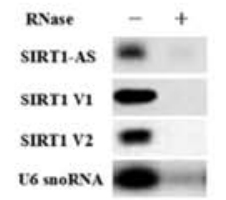

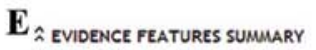

\begin{tabular}{|c|l|l|l|}
\hline \multirow{3}{*}{ HOMOLOGY FEATURES } & HIT NUIA & 0 & \\
\cline { 2 - 4 } & HIT SCORE & 0.0 & \\
\cline { 2 - 4 } & FRAME SCORE & 0.0 & \\
\hline \multirow{3}{*}{ ORF_FRAMEFINDER } & COVERAGE & 13.375 & \\
\cline { 2 - 4 } & LOG-ODOS SCORE & 32.47 & \\
\cline { 2 - 3 } & TYPE & Full & \\
\hline \multirow{2}{*}{ legend: non-coding } & \multicolumn{2}{|l}{} \\
\hline
\end{tabular}

D 1 CAAGTAAAAAACACTGGTTTTGTATTTCAAAAGTGGAaGGAaGATATCCAGTCATTAAACAGTCTACAAA 70
PCR Formard primer
71 ACATATGCCAGTAAATTACATAAAAGACTATGTACAATATAAAAAGAGCTGAAAACAGTCTTCACTGTAA 140 141 AaATAATTTAAAACAAACTTTTCAATTTAAAATATCATCTATAGCACACAAACATCATGCAAATGGAAAA 21 211 CTAAATATACTGCATTCTTTAGTGTAGCCAAATAAATTCAGATTGAGACATCTTATAAGTAGGGAAATGG 280 281 CCATTCAATACGATTTTTTTCTCTGGCAGTAATGGTCCTAGCTGGGTGTTTTATGCATAAAGAACAGCTA 350 351 TATTTCAAACCCTTTTTATTGTAATAAATACTAAAGCAACAGAGGAATACTTTATTAATTTAGGAGTGAT 420 421 GTTCAAAAATGGTCTGAAAAATAAATGCTTACTGTGCAATTTCATAAAGTATGAACTTCTTGAAAGACTA 490 491 GAAGCTTTTGCAGCTGAGAAAGITGATCTCTAGTTTTAAGGCAGGCTAAGCTTTTAAATAAAGATAAATT 560 561 ATAAGAACTAGTTTCATTCATTAACTATTCTGCTATTACAAGTTACATCATCATGTTCATCTCCATAATA 630 The mutation site ( $622 \mathrm{P}>\mathrm{C})$ 631 CTAGGGCTAGCAGTTTGGTATTT WACACCAAATCCTCCCAATGGGAGACATGTACTITCTGGCTGAATTA 700 701 CAATTAACATCTTATTACTTTGGAACTGTATAATCAGGGGCCTGTTGCTCTCCTCATTAATGG 763

Figure 1. SIRT1 locus causes a single-exon AS transcript. (A) Genomic landscape of SIRT1-AS. SIRT1-AS overlaps with SIRT1 mRNA 3'UTR; SIRT1 mRNA variant 1 and 2 are identical with the exception of the first exon, the arrows indicate the transcriptional direction, the purple rectangles show the cds region and the gray ones show the UTR. (B) Identification of SIRT1-AS by strand-specific RT-PCR. RNA samples digested by DNase I were added in different reaction systems as follows: ssRT, strand-specific reverse transcription; nsRT, non-specific reverse transcription, as a positive control; control, adding common RNase A to digest the RNAs, as a negative control. The RT products or digestive residue were applied by PCR and the PCR products were checked on a $1.5 \%$ agarose gel with ethidium bromide staining. (C) Identification of SIRT1-AS by northern blotting. Specific probes for SIRT-AS, SIRT1 variant 1, SIRT1 variant 2 and U6 snRNA probe (as control) were applied to analyze $20 \mu \mathrm{g}$ of total RNA. (D) The sequence of SIRT1-AS acquired by high-precision sequencing. Important motifs and primers used in strand-specific RT-PCR are shown in various colors. (E) SIRT1-AS was predicted as a non-coding RNA by the Coding Potential Calculator.

to SIRT1-AS) at $37^{\circ} \mathrm{C}$ for $3 \mathrm{~h}$ under simulated physiological conditions (10 mM Tris- $\mathrm{HCl} \mathrm{pH} 7.4,15 \mathrm{mM} \mathrm{NaCl}$ ). Then, $20 \mathrm{U}$ RPA-grade RNase A (Applied Biosystems) was added to each mixture to remove the single-strand RNAs. Four microliters of each residue was added to the $20 \mu$ l volume double-strand reverse transcription reaction with $5 \mathrm{pM}$ random primers adding SuperScript III reverse transcriptase (both from Invitrogen-Life Technologies). The system was incubated at $60^{\circ} \mathrm{C}$ for $60 \mathrm{~min}$ and terminated at $75^{\circ} \mathrm{C}$ for $15 \mathrm{~min}$, and the resultant double-strand cDNAs were amplified in a $25 \mu \mathrm{l} \mathrm{PCR}$ reaction system as templates. The primers used were: forward, 5'-ATT CTG CTA TTA CAA GTT-3' and reverse, AAG TAC ATG TCT CCC AT. After a 35-cycle amplification, the product was checked by electrophoresis on a $1.5 \%$ agarose gel with ethidium bromide staining.

Population investigated. In the in vivo study of the association between SIRT1-AS level with HCC risk, 52 patients (26 male and 26 female; average age $49.2 \pm 18.3$ years) were invited to participate in the study. The patients were diagnosed as primary HCC and treated with tumor resection in our hospital from May, 2012 to June, 2014. The patients did not suffer any complications. Their tumor sizes ranged from 3 to $6 \mathrm{~cm}$. There was no angiogenesis and invasion to peripheral tissues. Twenty-six male and 26 female healthy volunteers (average age $49.4 \pm 18.6$ years) also participated in the investigation. Each individual in the control group was gender- and age-matched with the patients in a one-on-one manner.

The study protocol was approved by the Ethics Committee of The Affiliated Hospital of Inner Mongolia Medical College.
The subjects provided written informed consent prior to their inclusion in the study.

Calculation of odds ratio $(O R)$. The odds ratio was used as a precise estimation for the risk of $622 \mathrm{U}>\mathrm{C}$ mutation in SIRT1-AS contributing to HCC in this study. The 2,000 primary HCC patients and 1,500 volunteers were from The Inner Mongolia Autonomous Region and Northeast China. Male and female subjects were equally divided, and had an age ranged of 22-58 years. Among the 2,000 HCC patients, the proportion of each stage (HCC staging according to the TNM Standard published by the Union for International Cancer Control in 2003) was approximately the same. The odd ratio was calculated as: Odd ratio $\mathrm{T}=$ (number of patients possessing allele $\mathrm{T}$ $\mathrm{x}$ number of volunteers possessing allele $\mathrm{C}$ )/(number of patients possessing allele $\mathrm{C} x$ number of volunteers possessing allele $\mathrm{T}$ ), and the odd ratio $\mathrm{C}=$ (number of patients possessing allele $\mathrm{C} \mathrm{x}$ number of volunteers possessing allele $\mathrm{T}$ )/(number of patients possessing allele $\mathrm{T} x$ number of volunteers possessing allele $\mathrm{C}$ ).

Website references. SIRT1-AS was predicted using information obtained from the Natural Antisense Transcript Database (http://natsdb.cbi.pku.edu.cn). SIRT1 mRNA variant sequences were obtained from GenBank (http://www.ncbi.nlm.nih.gov) and UCSC Genome (http://genome.ucsc.edu/index.html). The Coding Potential Calculator (http://cpc.cbi.pku.edu.cn/) was used to calculate the coding potential of the sequences. The secondary structures of WT SIRT1-AS and the mutant were predicted by the mFold online server (http://mfold.rna.albany. edu/?q=mfold). 
A

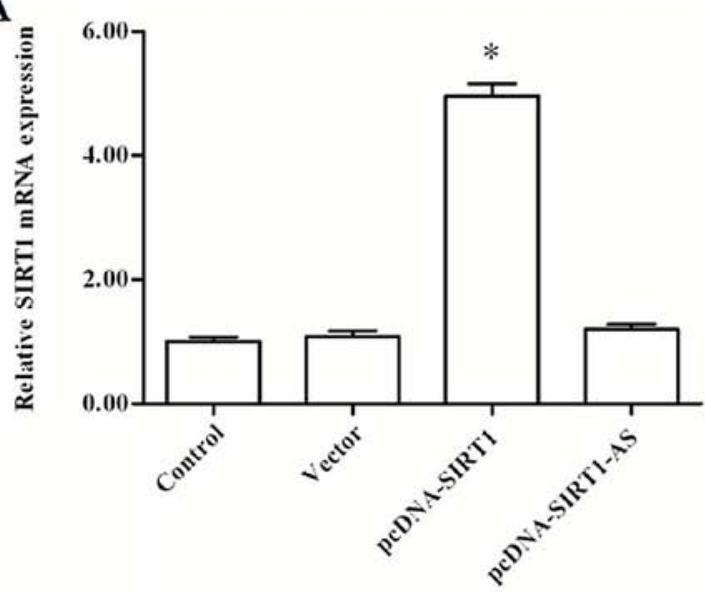

C

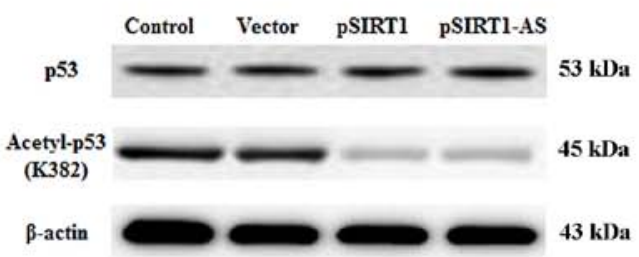

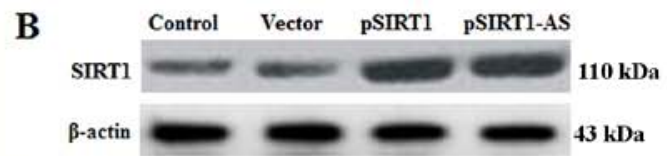
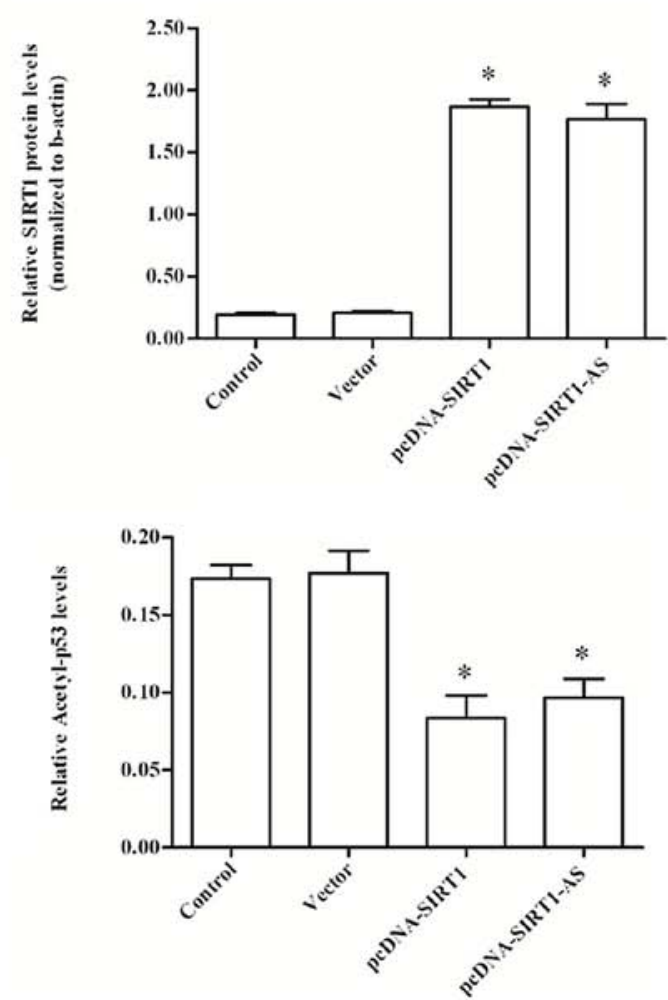

D
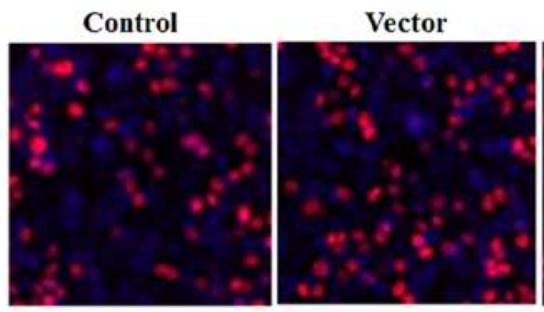

pCDNA-SIRT1

pCDNA-SIRT1 AS
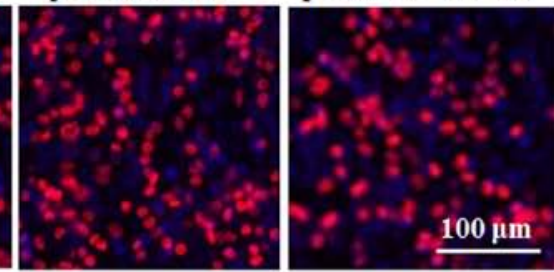

$\mathbf{E}$ $\rightarrow$ Control $\rightarrow$ Vector $\rightarrow$ peDNA-SIRTI $\rightarrow$ peDNA-SIRTI AS

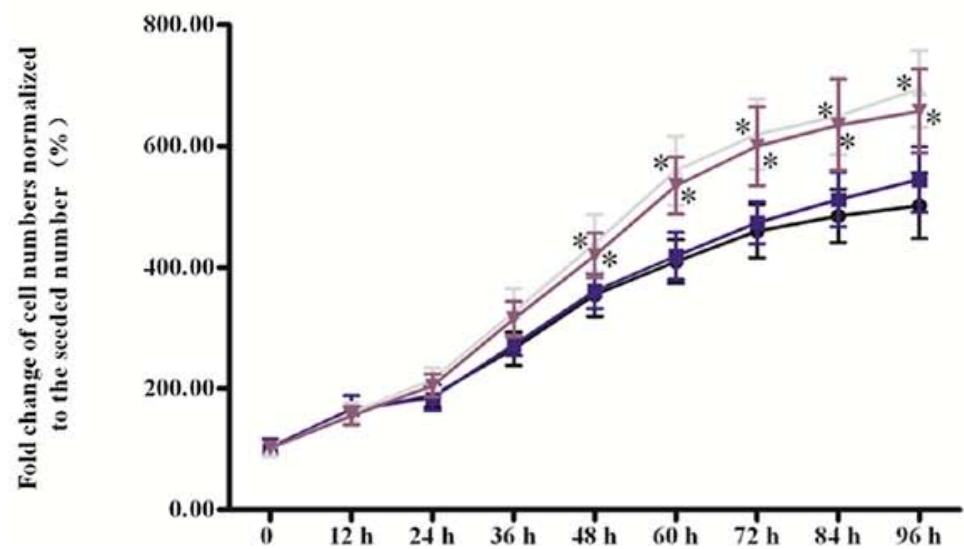

Figure 2. Overexpression of SIRT1-AS elevated SIRT1 protein level and promotion of the proliferation of HCC-9903 cells. Expression of (A) SIRT1 mRNA and (B) SIRT1 protein in HCC-9903 cells following the transfection of pcDNA-SIRT1 or pcDNA-SIRT1-AS for $48 \mathrm{~h}$. The mRNA and protein levels were normalized to $\beta$-actin. (C) Overexpression of SIRT1 or SIRT1-AS reduced the phosphorylated p53. (D) Detection of HCC-9903 proliferation by Edu approach (magnification, x200). After transfection of pcDNA constructs for $48 \mathrm{~h}$, cell proliferation was detected using Edu (red) mothed. The nuclei were stained by Hoechst (blue). The length of the bar is $2 \mathrm{~cm}$. (E) Changes of HCC-9903 cell viability after overexpression of SIRT1 or SIRT1-AS. HCC-9903 cells (1x105) were seeded in each 6-well plate. After $24 \mathrm{~h}$, pcDNA-SIRT1 or pcDNA-SIRT1-AS was transfected into the cells. Cell viability was examined every $12 \mathrm{~h}$ using the CellTiter-Blue H cell viability assay kit until $96 \mathrm{~h}$. Cell proliferation during the $96 \mathrm{~h}$ was exhibited by the fold change of cell numbers. The cell numbers were normalized to those originally seeded. ${ }^{*} \mathrm{P}<0.05$. HCC, hepatocellular carcinoma. 
A

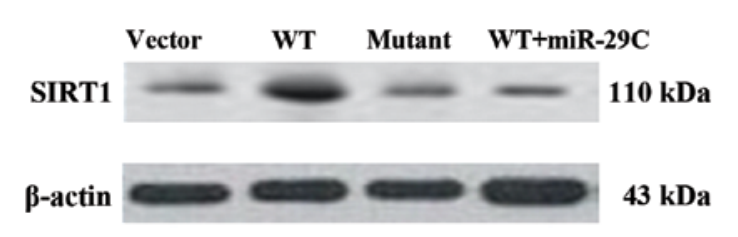

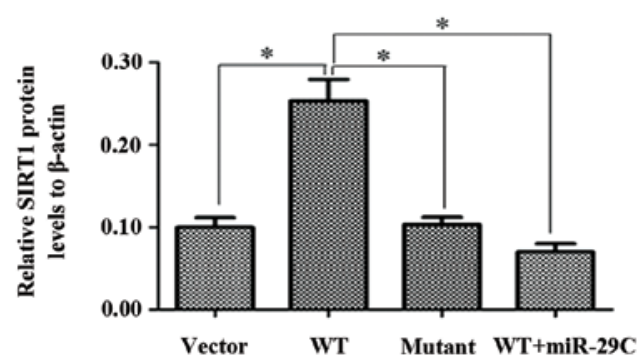

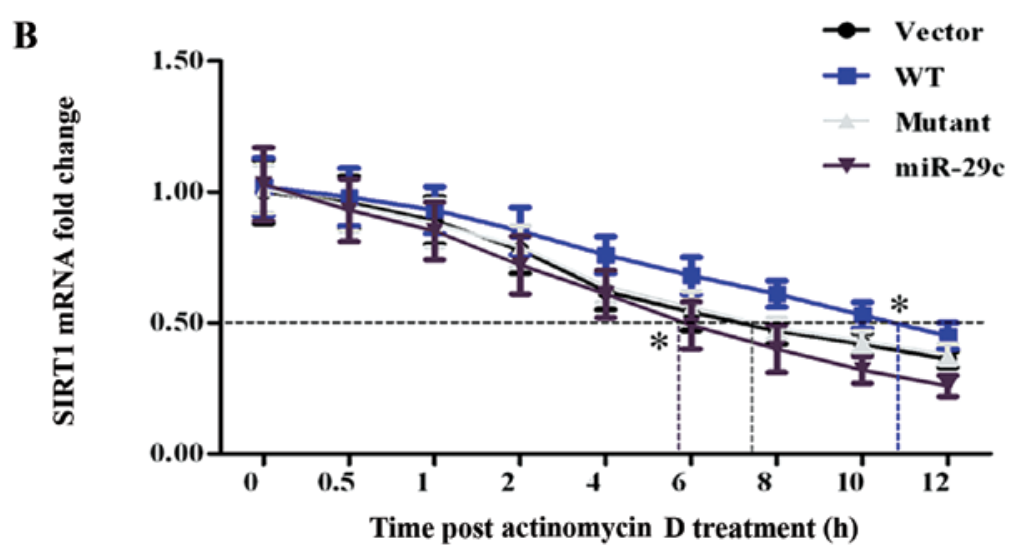

Figure 3. The 622C mutation disables SIRT1-AS to stabilize SIRT1 mRNA. (A)No. 622U>C mutant SIRT1-AS did not elevate the SIRT1 protein level. HCC9903 cell were transfected with negative vector, pcDNA-WT-SIRT1-AS, pcDNA-mutant-SIRT1-AS, or pcDNA-WT-SIRT1-AS and miR-29c mimic for $48 \mathrm{~h}$. The expression of SIRT1 protein was then examined. (B) WT SIRT1-AS prolonged the half-life of SIRT1 mRNA although the mutant did not. After $48 \mathrm{~h}$ of transfection of each construct or miR-29c mimic, HCC-9903 cells were treated with $2 \mu \mathrm{g} / \mathrm{ml}$ actinomycin D to inhibit transcription. The cells were harvested at $0,0.5,1,2,4,6,8,10$ and $12 \mathrm{~h}$ after treatment, and the residual mRNAs were measured by RT-qPCR. GAPDH mRNA was applied as an internal control, which was relatively stable within $32 \mathrm{~h}$. Stabilization of SIRT1 mRNA was weight by the time of half-life. ${ }^{*} \mathrm{P}<0.05$. HCC, hepatocellular carcinoma.

Statistical analysis. Data were obtained from at least three independent experiments. Values were presented as means \pm SEM. Statistics were calculated using SPSS statistics v19.0 software. Multiple comparisons were assessed by one-way ANOVA followed by Dunnett's tests. The difference between groups was considered statistically significant when $\mathrm{P}<0.05$.

\section{Results}

Identification of single-exon SIRT1-AS overlapping with SIRT1 3'UTR. We detected part of a transcript derived from the antisense strand of the SIRT1 3'UTR region by specific-strand RT-PCR in RNA samples that were extracted from the HCC-9903 cell line (Fig. 1B). Then, a specific probe was applied in the northern blotting to assay the transcript and a single band was obtained (Fig. 1A and C). The probed AS transcript was extracted and sent to BGI (Shenzhen, China) for high-precision sequencing. The sequencing results revealed the single-exon AS transcript with a size of $763 \mathrm{nt}$, designated as SIRT1-AS (Fig. 1D). The sequence was inserted into a coding potential calculator to calculate its coding potential. The outputs suggested that SIRT1-AS was probably non-protein-coding, because no available ORF structure was identified in the sequence (Fig. 1E). Therefore, we identified a single-exon AS transcript that overlapped with SIRT1 3'UTR and it was probably an AS lncRNA.

SIRT1-AS promotes the proliferation of HCC-9903 cells by upregulating the SIRT1 protein level. The pcDNA-SIRT1-AS or pcDNA-SIRT1 expression vectors were transfected into HCC-9903 cells to assess the role of SIRT1-AS in HCC cell proliferation. The specific-strand RT-qPCR and western blotting results showed that SIRT1-AS overexpression did not influence SIRT1 mRNA expression, but markedly elevated the SIRT1 protein level (Fig. 2A and B). As a result, the downstream anti-oncogene p53 was deacetylated by SIRT1 and SIRT1-AS overexpression (Fig. 2C). Edu staining and cell viability detection by the CellTiter-Blue $\mathrm{H}$ cell viability assay kit revealed that SIRT1 and SIRT1-AS overexpression promoted the proliferation of HCC-9903 cells (Fig. 2D and E). The results suggested that SIRT1-AS functioned positively in the regulation of SIRT1 expression at the post-transcriptional level.

The 622C single-nucleotide mutation disables SIRT1-AS to stabilize SIRT1 mRNA. In clinical diagnosis, our team identified a single-nucleotide mutation in a small number of HCC patient's SIRT1-AS by gene sequencing assistant diagnosis. The no. 622 base T (also U in RNA) was substituted by C in the minority of the patients (the site was marked in Fig. 1D). To verify the performance of the mutant SIRT1-AS, a pcDNA-SIRT1 mutant was additionally established. WT or mutant SIRT1-AS expression constructs were transfected into HCC-9903 cells. After an incubation for $48 \mathrm{~h}$, western blot assay showed that the SIRT1 protein level was elevated in the WT transfection group, but did not significantly change in the group of mutant SIRT1-AS transfection (Fig. 3A). Additionally, as a proven SIRT1-targeting miRNA, miR-29c mimics reduced the protein level that was elevated by WT SIRT1-AS (Fig. 3A). 
$\mathbf{A}$

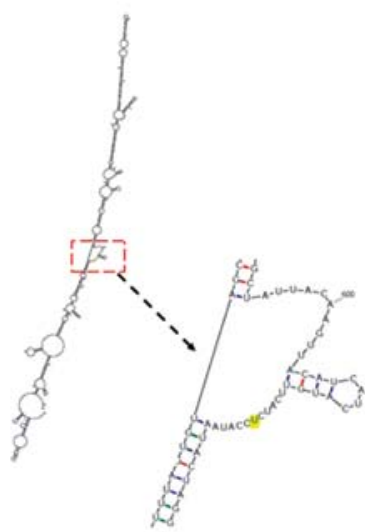

WT SIRT1-AS (622U)

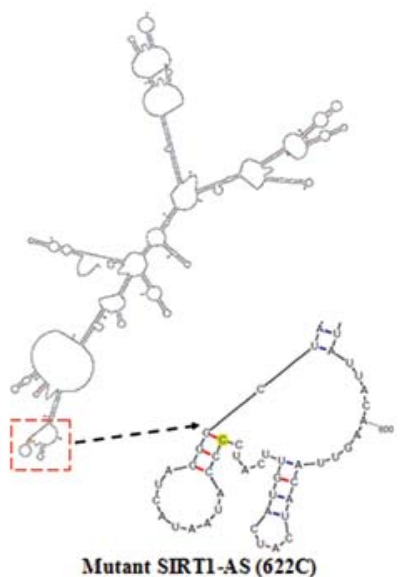

Mutant SIRTI-AS (622C)

B

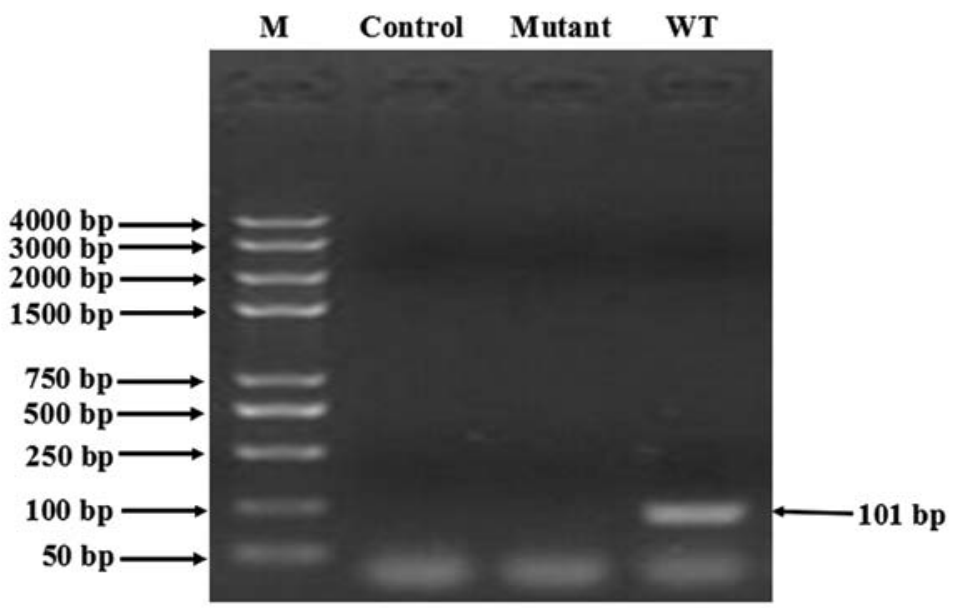

Figure 4. The 622C mutation disables SIRT1-AS to mask the miR-29c binding site in SIRT1 mRNA 3'UTR. (A) The 622U>C mutation probably resulted in a marked alteration in the SIRT1 mRNA secondary structure. Sequences of WT (622U, left) and the mutant (622C, right) SIRT1-AS were put into the mFold web server to calculate their optimal secondary structure, a the mutant site was highlighted yellow. (B) The 622C mutation disabled SIRT1-AS to bind with SIRT1 mRNA around the miR-29c binding site. WT or the mutant synthetic SIRT1-AS was respectively incubated with their complementary SIRT1 mRNA fragment under simulated physiological conditions for $3 \mathrm{~h}$, with single-strand SIRT1 mRNA only serving as the control. The mixture was digested by RPAgrade RNase A. RT-PCR was performed to detect whether an RNA duplex was formed. The final products were checked on a $1.5 \%$ agarose gel with ethidium bromide staining. RPA, ribonuclease protection assay.

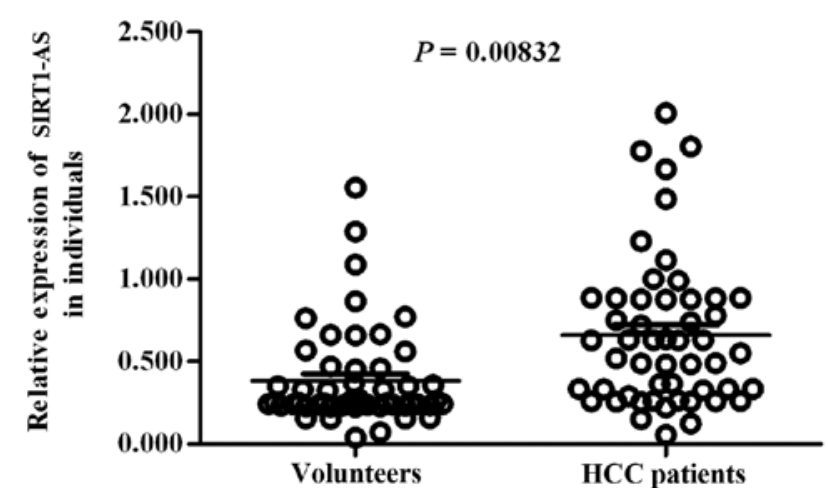

Figure 5. SIRT1 is more highly expressed in liver tissues of HCC patients than non-hepatopathic people. Strand-specific RT-qPCR was performed to examine the total SIRT1-AS levels in the liver tissues of $52 \mathrm{HCC}$ patients and 52 non-hepatopathic volunteers. The mRNA levels were normalized to individual $\beta$-actin mRNA. The significant level was set at $\mathrm{P}<0.01$. HCC, hepatocellular carcinoma.

The transfected cells were treated with $2 \mu \mathrm{g} / \mathrm{ml}$ actinomycin D to block the transcription activity in the cells. After treatment for $0,0.5,1,2,4,6,8,10$ and $12 \mathrm{~h}$, the cells were collected and used to extract total RNA separately. The residual SIRT1 mRNA was quantified to evaluate the variation of the half-life between the groups. The results revealed that the half-life of SIRT1 mRNA was $\sim 8 \mathrm{~h}$ under normal circumstances, which was not influenced by the mutant. As expected, the miR-29c mimic significantly shortened the half-life of SIRT1 mRNA (to $<6$ h, Fig. 3B). Notably, the WT SIRT1-AS greatly extended the period to $\sim 11 \mathrm{~h}$ (Fig. 3B). In particular, WT SIRT1-AS was likely to antagonize the negative regulation of miR-29c.

WT SIRT1-AS masked the miR-29c binding site on the 3'UTR of SIRT1 mRNA whereas the mutant did not mask the binding site. As the miR-29c binding site was within the overlapping region of SIRT1-AS and SIRT1 mRNA, we hypothesized that WT SIRT1-AS was able to bind to SIRT1 mRNA, thus miR-29c was unable to target SIRT1 mRNA, whereas the mutant was not able to bind to SIRT1 mRNA. The sequences of WT SIRT1-AS and the mutant were, respectively, entered into the mFold web server to calculate the most probable secondary structures. The outputs showed that their secondary 
Table I. Investigation of different genotypes in HCC patients and non-hepatopathy volunteers.

\begin{tabular}{lcrc}
\hline & \multicolumn{2}{c}{ Genotype } & \\
\cline { 2 - 3 } & $\begin{array}{c}\text { WT } \\
\text { Variables }\end{array}$ & $\begin{array}{c}\text { Mutant } \\
(622 \mathrm{C})\end{array}$ & $\begin{array}{c}\text { Mutation ratio } \\
(\%)\end{array}$ \\
\hline HCC patients & 1906 & 94 & 4.8 \\
Volunteers & 1255 & 245 & 16.3 \\
Odds ratio & 3.958 & 0.253 & \\
\hline
\end{tabular}

HCC, hepatocellular carcinoma.

structures were entirely different (Fig. 4A), suggesting at their distinct ability to combine with SIRT1 mRNA. WT or the mutant synthetic SIRT1-AS was incubated with SIRT1 mRNA under simulated physiological conditions for $3 \mathrm{~h}$, and then the mixture was digested with RPA-grade RNase A. RT-PCR was subsequently performed to detect whether an RNA duplex was formed. A 101-bp band, which included the mutant site and cDNA of the miR-29c binding site, was only detected in the residue of the WT SIRT1-AS/mRNA mixture. The RPA results showed that only WT SIRT1-AS formed a sense-antisense RNA duplex (Fig. 4B), which also supported our hypothesis.

Mutation decreases the risk of HCC. The above results suggested that the mutation $622 \mathrm{U}>\mathrm{C}$ 're-liberated' the miR-29c binding site via WT SIRT1-AS and downregulated SIRT1 expression at the post-transcriptional level. We randomly detected total SIRT1-AS levels in the liver tissues of 52 HCC patients and 52 non-hepatopathic volunteers. The strand-specific RT-qPCR results showed that SIRT1-AS was more highly expressed in HCC patients (Fig. 5). A survey was performed to assess the relationship between $622 \mathrm{C}$ and the risk of HCC. Among 2,000 HCC patients, the mutant ratio was $4.8 \%$ smaller than that among 1,500 non-hepatopathic volunteers (up to $16.3 \%$ ). The statistical analysis showed that the OR value of $622 \mathrm{C}$ was only $0.253(<1)$, which was lower than that of $622 \mathrm{~T}(3.958>1)$ (Table I). These results suggested that the mutant $\mathrm{C}$ allele reduced the risk of $\mathrm{HCC}$.

\section{Discussion}

In the present study, we characterized the AS lncRNA SIRT1-AS which suppressed the miRNA-induced translational repression of SIRT1 mRNA by masking the miR-29c binding site on the SIRT1 3'UTR. In vitro studies showed that SIRT1-AS positively regulates HCC proliferation, and the comparison between the HCC patients and non-hepatopathic volunteers indicated that high-level expression of the WT SIRT1-AS increases the risk of HCC. Additionally, we identified a 622U>C mutant of SIRT1-AS in the clinical diagnosis of HCC. The in vitro experiments and statistical analysis of sequences strongly suggested that the mutation 're-liberated' the masked miR-29c binding site and contributed to decreasing the risk of HCC.
Few lncRNAs have been found to be dysfunctional in HCC; however, to the best of our knowledge, none of them were transcribed from the AS strand of a functional gene (20). Thus, our results present the first identification of a p53-repressing AS lncRNA acting as a carcinogenic factor. Half of the human genomic loci lead to NATs; however, NATs associated with $\mathrm{HCC}$ or other liver cancers have rarely been reported. It is expected that NATs associated with HCC or other liver cancer types are a promising field in liver cancer biology.

Gene regulation by AS transcription is complicated. AS lncRNAs that share apparently identical mechanisms often have opposite functions. BACE1-AS IncRNA and tie-1 AS lncRNA formed a sense/antisense RNA duplex with their mRNAs. Of note, BACE-AS IncRNA masked miR-485-5p binding site in the mRNA and stabilized BACE1 mRNA, while tie-1 AS lncRNA negatively regulated tie-1 expression by downregulating tie-1 mRNA $(21,22)$. A negative or positive response may depend on the site where the S-AS is bound and the binding length of the two transcripts. In the present study, SIRT1-AS functioned in a similar manner to BACE1-AS in their sense gene regulation. The RPA results exhibited the binding region of WT SIRT1-AS, and SIRT1 mRNA included the binding site of the SIRT1 suppressor miR-29c. The mutation resulted in SIRT1-AS being unable to bind to SIRT1 mRNA and mask the miR-29c binding site. Therefore, the mutant SIRT1-AS played a negative role in SIRT1 translation.

In the clinical context, there is a need for the verification of therapeutic targets for HCC. The mutation 622C in SIRT1-AS decreased the risk of HCC, thus the mutant may be regarded as a potential target for gene therapy for $\mathrm{HCC}$ or other diseases associated with SIRT1 dysregulation.

In conclusion, we characterized a carcinogenic NAT (also known as AS IncRNA) SIRT1-AS and identified a benign mutation $622 \mathrm{U}>\mathrm{C}$ in its sequence. An in vitro study and statistical analysis indicated that the mutation minimized the risk of HCC. The findings of this study provide a new therapeutic target for HCC and improve our understanding of the mechanisms of AS lncRNAs.

\section{References}

1. Werner A and Berdal A: Natural antisense transcripts: Sound or silence? Physiol Genomics 23: 125-131, 2005.

2. He Y, Vogelstein B, Velculescu VE, Papadopoulos N and Kinzler KW: The antisense transcriptomes of human cells. Science 322: 1855-1857, 2008.

3. Katayama S, Tomaru Y, Kasukawa T, Waki K, Nakanishi M, Nakamura M, Nishida H, Yap CC, Suzuki M, Kawai J, et al; FANTOM Consortium: Antisense transcription in the mammalian transcriptome. Science 309: 1564-1566, 2005.

4. Faghihi MA and Wahlestedt C: Regulatory roles of natural antisense transcripts. Nat Rev Mol Cell Biol 10: 637-643, 2009.

5. Pelechano V and Steinmetz LM: Gene regulation by antisense transcription. Nat Rev Genet 14: 880-893, 2013.

6. Magistri M, Faghihi MA, St Laurent G III and Wahlestedt C: Regulation of chromatin structure by long noncoding RNAs: Focus on natural antisense transcripts. Trends Genet 28: 389-396, 2012.

7. Bertozzi D, Iurlaro R, Sordet O, Marinello J, Zaffaroni N and Capranico G: Characterization of novel antisense HIF-1 $\alpha$ transcripts in human cancers. Cell Cycle 10: 3189-3197, 2011.

8. Bhan A, Hussain I, Ansari KI, Kasiri S, Bashyal A and Mandal SS: Antisense transcript long noncoding RNA (lncRNA) HOTAIR is transcriptionally induced by estradiol. J Mol Biol 425: 3707-3722, 2013. 
9. Su WY, Li JT, Cui Y, Hong J, Du W, Wang YC, Lin YW, Xiong $\mathrm{H}$, Wang JL, Kong X, et al: Bidirectional regulation between WDR83 and its natural antisense transcript DHPS in gastric cancer. Cell Res 22: 1374-1389, 2012.

10. Haigis MC and Guarente LP: Mammalian sirtuins - emerging roles in physiology, aging, and calorie restriction. Genes Dev 20: 2913-2921, 2006.

11. Michan S and Sinclair D: Sirtuins in mammals: Insights into their biological function. Biochem J 404: 1-13, 2007.

12. Finkel T, Deng CX and Mostoslavsky R: Recent progress in the biology and physiology of sirtuins. Nature 460: 587-591, 2009.

13. Chang HC and Guarente L: SIRT1 and other sirtuins in metabolism. Trends Endocrinol Metab 25: 138-145, 2014.

14. Lagouge M, Argmann C, Gerhart-Hines Z, Meziane H, Lerin C, Daussin F, Messadeq N, Milne J, Lambert P, Elliott P, et al: Resveratrol improves mitochondrial function and protects against metabolic disease by activating SIRT1 and PGC-1 $\alpha$. Cell 127: 1109-1122, 2006.

15. Yeung F, Hoberg JE, Ramsey CS, Keller MD, Jones DR, Frye RA and Mayo MW: Modulation of NF-kappaB-dependent transcription and cell survival by the SIRT1 deacetylase. EMBO J 23: 2369-2380, 2004.

16. Bai L, Pang WJ, Yang YJ and Yang GS: Modulation of Sirt1 by resveratrol and nicotinamide alters proliferation and differentiation of pig preadipocytes. Mol Cell Biochem 307: 129-140, 2008.
17. Cheng HL, Mostoslavsky R, Saito S, Manis JP, Gu Y, Patel P, Bronson R, Appella E, Alt FW and Chua KF: Developmental defects and p53 hyperacetylation in Sir2 homolog (SIRT1)-deficient mice. Proc Natl Acad Sci USA 100: 10794-10799, 2003.

18. Lee JT and Gu W: SIRT1: Regulator of p53 deacetylation. Genes Cancer 4: 112-117, 2013.

19. Bae HJ, Noh JH, Kim JK, Eun JW, Jung KH, Kim MG, Chang YG, Shen Q, Kim SJ, Park WS, et al: MicroRNA-29c functions as a tumor suppressor by direct targeting oncogenic SIRT1 in hepatocellular carcinoma. Oncogene 33: 2557-2567, 2014.

20. He Y, Meng XM, Huang C, Wu BM, Zhang L, Lv XW and Li J: Long noncoding RNAs: Novel insights into hepatocellular carcinoma. Cancer Lett 344: 20-27, 2014.

21. Faghihi MA, Modarresi F, Khalil AM, Wood DE, Sahagan BG, Morgan TE, Finch CE, St Laurent G III, Kenny PJ and Wahlestedt C: Expression of a noncoding RNA is elevated in Alzheimer's disease and drives rapid feed-forward regulation of $\beta$-secretase. Nat Med 14: 723-730, 2008.

22. Li K, Blum Y, Verma A, Liu Z, Pramanik K, Leigh NR, Chun CZ, Samant GV, Zhao B, Garnaas MK, et al: A noncoding antisense RNA in tie-1 locus regulates tie-1 function in vivo. Blood 115: 133-139, 2010. 